Relations industrielles

Industrial Relations

\title{
Managing by Communication, by Willard V. Merrihue, McGraw-Hill Co. Inc., 1960, 300 pp., \$7.05.
}

\section{Louis-Marie Tremblay}

Volume 15, numéro 2, avril 1960

URI : https://id.erudit.org/iderudit/1022045ar

DOI : https://doi.org/10.7202/1022045ar

Aller au sommaire du numéro

Éditeur(s)

Département des relations industrielles de l’Université Laval

ISSN

0034-379X (imprimé)

1703-8138 (numérique)

Découvrir la revue

Citer ce compte rendu

Tremblay, L.-M. (1960). Compte rendu de [Managing by Communication, by Willard V. Merrihue, McGraw-Hill Co. Inc., 1960, 300 pp., \$7.05.] Relations industrielles / Industrial Relations, 15(2), 280-281.

https://doi.org/10.7202/1022045ar

Tous droits réservés (C Département des relations industrielles de l’Université Laval, 1960
Ce document est protégé par la loi sur le droit d'auteur. L’utilisation des services d'Érudit (y compris la reproduction) est assujettie à sa politique d'utilisation que vous pouvez consulter en ligne.

https://apropos.erudit.org/fr/usagers/politique-dutilisation/ 
En employant la classification de Hoxie, nous dirions qu'il doit cesser d'être un mouvement «up lift » ou «predatory» pour devenir «businesslike ».

\section{Ls-Marie Tremblay}

Le travail selon saint Jean Chrysostome, par Lucien Daloz, P. Lethielleux, coll. «Théologie, pastorale, spiritualité », Paris, 1959, 1 vol., 194 pp.

Il y a quelques années, dans un article qu'il écrivait pour la revue Esprit, lequel a été ensuite réédité sous le titre Pour une théologie du travail, le R.P. Chenu observait, non sans amertume, que l'on n'était pas encore parvenu à élaborer une théologie du travail alors que la réflexion des théologiens depuis longtemps s'était portée sur des sujets comme la guerre, les affaires, l'histoire, etc. Pourtant, ce n'est pas le besoin qui manque. Car le travail dans notre civilisation a acquis une signification et une importance considérable. Et puis des erreurs d'optique très graves sont en train de se frayer un chemin dans beaucoup d'esprits.

Si une véritable théologie du travail n'a pas été élaborée, les chrétiens ont toujours eu des vues sur la nature du travail, sa place et son rôle dans la vie. En ces derniers temps, il faut se réjouir de voir des théologiens se pencher sur ce sujet et mener des recherches systématiques conduisant à une véritable théologie du travail.

L'ouvrage que présente Lucien Daloz est une heureuse contribution dans ce domaine. Il est vrai qu'il se limite à un secteur particulier de la Tradition, mais une multitude d'études semblables sont nécessaires si l'on veut en arriver à une oeuvre de synthèse.

L'auteur a donc relevé dans les écrits de saint Jean Chrysostome et les Pères Antiochiens ce qu'ils ont dit sur le travail. Si aucun d'eux n'a traité à fond ce problème, il leur est arrivé souvent d'exprimer ici et là comment ils le concevaient.

Voici le titre des chapitres sous lesquels l'auteur analyse leurs réflexions: L'homme dans le monde matériel; la dignité du travail; le travail dans l'histoire du salut; travail et nature humaine; travail et relations humaines; la technè; Dieu et le travail de l'homme; le travail dans la tradition antiochienne; conclusions.

Nous ne saurions dire tout l'intérêt que nous avons eu à lire cet ouvrage bien fait selon une méthode rigoureuse. Nous croyons que l'auteur a apporté une contribution indispensable à ceux qui voudront continuer d'explorer un tel sujet et ainsi collaborer à l'édification d'une théologie du travail.

GÉrard Dion

Managing by Communication, by Willard V. Merrihue, McGraw-Hill Co. Inc., 1960, 300 pp., \$7.05.

Voici l'un des trop rares bons volumes en provenance d'administrateurs qualifiés. A l'emploi de la General Electric, monsieur Merrihue a été le grand responsable de la mise en opération des programmes d'entraînement de cette compagnie en matière de communications et de relations industrielles. Dans le travail qu'il présente, il s'inspire de sa propre expérience et illustre constamment sa pensée par des exemples tirés en majeure partie des dossiers de cette compagnie.

Toutefois, Managing by Communication est un livre d'un intérêt limité qui s'adresse à un groupe particulier. Il apporte peu de nouveau et se situe surtout au niveau de généralités déjà connues. Parfois, la présentation et le ton so rapproche trop du «Comment se faire des amis » de Dale Carnegie. En ajoutant à la construction, l'absence de références et de notions bibliographiques, il présente peu d'intérêt sur le plan académique. D'ailleurs, l'auteur ne lui assigne pas cette fin.

Dans la préface il écrit: «This book has been designed to help managers at all levels from first line supervision to chief executives better to understand how to get work performed through people». «There has been no intent to delve deeply into communication theory, nor to write as a theorician or specialist in the complex social, political and economic areas treated; rather the concern has been with the practical 
uses of the communication process within the business setting ». Cette optique limite le public auquel il s'adresse.

L'aspect le plus innovateur consiste dans la façon dont l'auteur conçoit les communications et la place qu'il leur accorde dans le travail de l'administrateur. Il en fait la fonction essentielle.

« Today's developing concept of leadership, exercised largely by persuasion rather than by command, places a great new premium on communication. Three of the elements of a manager's work, planning, organizing and measuring, are heavily dependent on employee communication: the fourth category, integration, is almost synonymous with communication 》 (p. 30). Plus loin, il ajoute: «Communication, is not a constricted concept of getting work performed by people through listening and talking and writing to them. Neither is it a concept of manipulating people through hypnotic semantics. Rather, communication is a process which both affects and is affected by the deeds and attitudes of management, on one hand, and the employees and public on the other. It is essentially an interpretive process. This implies that the successful business communicator - like the successful translator of languages must not only know the languages of both the senders and the receivers, but must also have a sympathetic understanding of the backgrounds, the attitudes and aspirations of both. Communication, then, is a broad conception equaling, in its magnitude and importance to the business, such other accepted or upcoming practices as automation, decentralization or operations research and synthesis 》 (p. 36).

C'est une conception très compréhensive de la fonction communication. L'auteur ne la perd jamais de vue dans les techniques qu'il propose. Il y revient même à plusieurs reprises pour rappeler que ce procédé d'interprétation est dynamique, qu'il est instrumental et non une fin en lui-même. "Communication is a mean - and a very effective mean - for the solution of managerial problems and for the attainment of managerial objectives » (p. 235).

Mais l'auteur amène, élabore, décrit des techniques et des principes avec parfois une confiance naïve dans leur efficacité ou leur capacité de solutionner à peu près tous les problèmes de la direction avec les employés, les syndicats et la communauté en général. Nul doute que ce livre peut aider à améliorer la productivité et la satisfaction au travail, à créer un climat plus réceptif devant l'innovation et à établir des conditions qui favorisent la compréhension mutuelle. Mais nous tenons à mettre le lecteur en garde contre l'impression qui se dégage à certains moments à l'effet qu'une recette éprouvée dans un certain milieu sera nécessairement valable ailleurs. Au contraire, ces recettes ne sont pas des arguments d'autorité, mais des exemples de ce qu'un peu d'imagination et de bonne volonté peuvent réaliser.

En résumé, ce livre comporte cinq parties d'inégale valeur ou importance mais d'un intérêt réel. La première partie porte sur la nature des communications; la seconde illustre comment l'administrateur doit utiliser les communications pour améliorer ses relations avec les employés, le syndicat et la communauté; la troisième traite $\mathrm{du}$ rôle du fonctionnaire en position d'autorité, spécialement du contremaître: la quatrième déorit un nombre imposant de techniques. principes et moyens de communications (orales - écrites - vers le haut, vers le bas, etc.); la cinquième enfin applique l'usage des communications à certaines fins spécifiques, telles que: les changements, les périodes de crises, les grèves, etc.

Somme tcute, un assez bon volume qui fait une synthèse de l'expérience actuelle et qui s'avérera utile pour celui qui est engagé dans l'administration.

Ls-Marie Tremblay

\section{Leadership, Psychology and Organiza- tional Behavior, by Bernard M. Bass, Harper \& Brothers, New York, 548 pp.}

Depuis quelques années l'on essaie de découvrir et de définir le meneur du groupe - «leader» - non plus par des traits individuels ou selon la théorie du grand homme mais surtout en fonction de la dynamique de groupe. En effet, ce qui justifie l'attribut de mereur c'est l'influence exercée sur le groupe. Plusieurs travaux ont été publiés récemment sur le développement 